\title{
Flexible Slow and Fast Light Using Tailored Brillouin Spectra in Optical Fibers
}

\author{
Luc Thévenaz, Sang-Hoon Chin, Kwang-Yong Song*, Miguel Gonzalez-Herraez** \\ EPFL, Swiss Federal Institute of Technology, STI-NAM Station 11, CH-1015 Lausanne \\ * Now with Dept. of Electronic Engineering, The University of Tokyo, Tokyo, Japan \\ ** On leave from Dept. of Electronics, University of Alcalá de Henares, Madrid 28805 Spain \\ Authore-mail address: Luc.Thevenaz@EPFL.CH
}

\begin{abstract}
Stimulated Brillouin scattering makes possible the generation of synthesized gain spectra, so that innovative slow light schemes can be realized, ranging from broadband tunable delays to a zero-gain situation identical to an ideal electromagnetically-induced transparency. (C)2006 Optical Society of America

OCIS codes: (999.9999) Slow light; (060.4370) Nonlinear optics, fibers; (290.5900) Scattering, stimulated Brillouin
\end{abstract}

\section{Introduction}

Slow light is known to be a very attractive approach to achieve all-optical delay lines and to provide a timing tool for photonics signal processing. A significant step towards real applications has been achieved last year when slow light was experimentally and efficiently demonstrated in optical fibres using stimulated Brillouin scattering [1] and later other nonlinear interactions $[2,3]$ with larger natural bandwidth but much reduced efficiency.

Actually stimulated Brillouin scattering (SBS) has proved to be an unprecedented and unmatched flexible tool for the generation of slow light regarding its spectral tailoring capability. Indeed, a large variety of gain spectral profile can be obtained by properly modulating the pump spectrum. When a monochromatic pump is used in the stimulated Brillouin interaction, the gain window appearing in the fiber transmission spectrum has a Lorentzian shape whose characteristic spectral FWHM width is around $30 \mathrm{MHz}$ in conventional single-mode fibers pumped at $1.55 \mu \mathrm{m}$. However, when the pump is modulated the gain bandwidth is given by the convolution of the pump spectrum and the Brillouin gain curve. Hence the effective Brillouin gain spectrum $g(\Delta v)$ is given by $g(\Delta v)=P(\Delta v) \otimes g_{B}(\Delta v)$ where $\otimes$ denotes convolution, $P(\Delta v)$ is the normalized pump power spectral density (i.e. its integral is unity) and $g_{B}(\Delta v)$ is the characteristic Lorentzian gain of the Brillouin amplification process.

The simplest synthesized pump spectrum is a two-frequency spectrum with a frequency separation from 0 to $50 \mathrm{MHz}$, in the range of the natural Brillouin linewidth [4]. This results in overlapping gain profiles and eventually a reversed linear phase variation, so that fast light in gain regime was achieved. This modulation scheme makes also possible varying delays by changing the frequency separation rather than the pump power, as shown in Fig.1. It can be a more convenient solution in many practical implementations, the pump power remaining steady and stable.

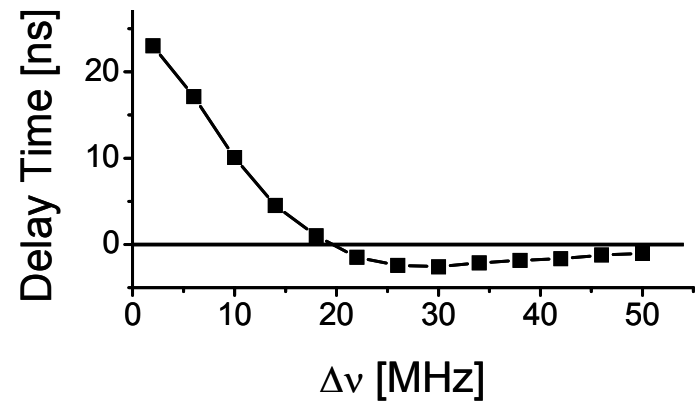

Fig.1 Measured delay time of the probe pulse as a function of the frequency difference $\Delta v$ between the 2 pump frequencies.

More recently it was demonstrated that the bandwidth of SBS-based slow light can be made arbitrarily large by actively broadening the pump spectrum using random direct current modulation of the pump laser [5]. A particularly useful case arises when the pump spectrum can also be approximated by a Lorentzian distribution. In such conditions, the effective Brillouin gain shape remains Lorentzian, but shows a width equal to the sum of the characteristic Brillouin gain width and the pump spectral width. Hence an adequate pump modulation can be used to broaden at will the Brillouin interaction, suppressing the inherent limitation related to the narrow natural linewidth. This scheme was then replicated and improved successfully to recently ultimately reach a $10 \mathrm{GHz}$ bandwidth [6]. 
All these slow-light techniques suffer from the drawback of a significant amplitude change associated with the delaying effect. For instance a one-pulse width delaying gives rise to a large $30 \mathrm{~dB}$ pulse amplitude change using SBS. We demonstrate here that the high flexibility of SBS opens unprecedented achievements and offers the possibility to synthesize a gain spectral profile, so that a signal delay or advance can be realized with an ideally absolute null amplitude change. This can be obtained by the combination of gain and loss spectral profiles with identical depth but different width, resulting in a net zero gain and a differential delaying effect.

For a gain/loss process following a Lorentzian spectral distribution such as SBS, a signal propagating in a medium showing a linear gain $G$ will experience a net amplitude change by a factor $\exp (G)$ together with a delay $T=G /(2 \pi \Delta v)$ where $\Delta v$ is the half width at half maximum of the Lorentzian distribution.

Now let superpose in the frequency domain a SBS gain with linear gain $+G_{l}$ and a bandwidth $\Delta v_{l}$ together with a SBS loss with linear negative gain (thus loss) $-G_{2}$ and a bandwidth $\Delta v_{2}$. The resulting linear gain is therefore $\mathrm{G}=G_{I^{-}}$ $G_{2}$ and the overall delay $T=G_{I} /\left(2 \pi \Delta v_{l}\right)-G_{2} /\left(2 \pi \Delta v_{2}\right)$. If $G_{I}=G_{2}$, then $G=0$ and $T=G_{1,2} / 2 \pi \times\left(\Delta v_{I}^{-1}-\Delta v_{2}^{-1}\right)$.

If the bandwidths of the gain and loss spectra are substantially different, e.g. $\Delta v_{2}>>\Delta v_{1}$, it is possible to obtain a significant delay $T$ with nevertheless a zero linear gain $G$. Fig. 2 shows the obtain gain/loss spectral profile, demonstrating that a good compensation of gain and loss can be obtained. We can achieve a situation identical to ideal electromagnetically-induced transparency (EIT), that is up to full transparency.
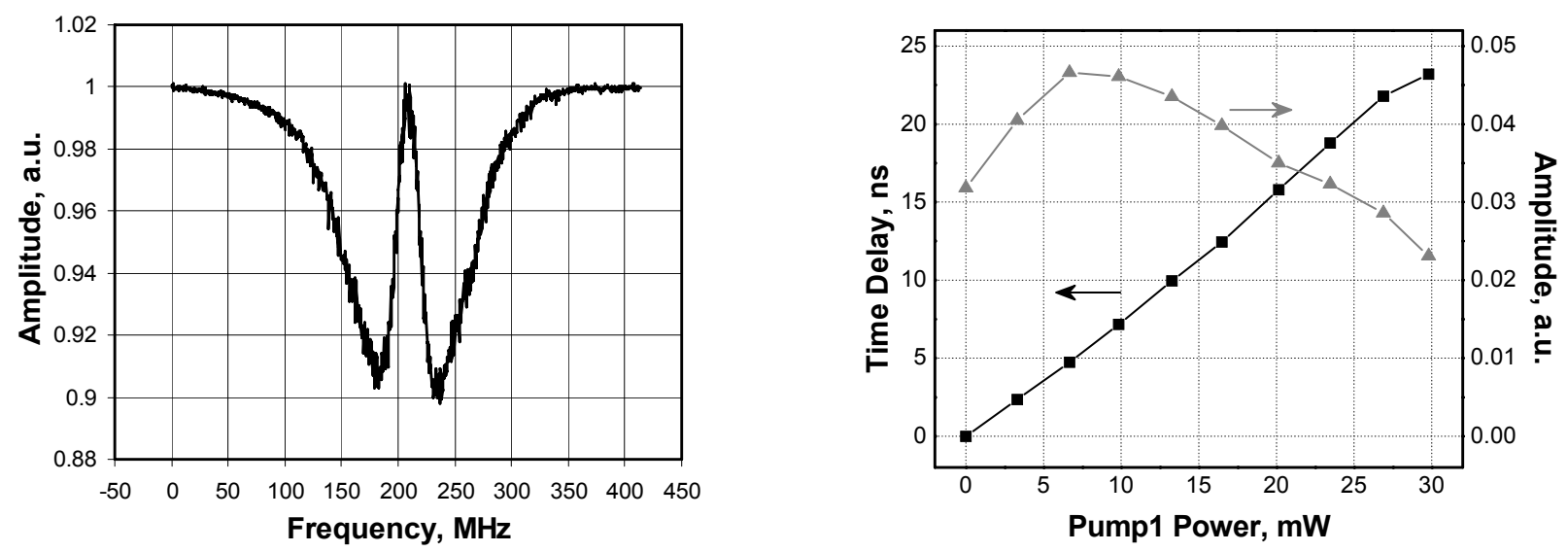

Fig.2: Left: Amplitude of a probe signal as a function of frequency after propagation in a $2 \mathrm{~km}$ fibre in which a superposed SBS gain/loss profile is generated. At the centre gain and loss fully compensate. Right: Measured delay and amplitude of a signal subject to the SBS gain spectrum shown on left, as a function of pump 1 power. The power of pump 2 is approx. 10 times higher than pump 1.

Delay and amplitude for a $1 \mathrm{MHz}$ sine modulated signal were recorded for different pump levels and are shown in Fig. 3 in linear scales. Delays comparable with the standard method [1] are obtained in a $2 \mathrm{~km}$ fiber, together with a maximum amplitude change of $\pm 1.5 \mathrm{~dB}$. An equivalent delay using the non-compensated standard technique would result in a $23 \mathrm{~dB}$ amplitude change.

These first results show that the ideal zero-gain situation is not exactly realized, explaining the amplitude growth for low pump power. The amplitude is then decreasing for higher power certainly as a consequence of pump depletion. We are confident to obtain even flatter amplitude response in the near future. It must be pointed out that the broadband gain compensation can be produced by other types of interactions, in particular Raman, but also using doped fibers and parametric amplification.

\section{References}

1 K. Y. Song, M. Gonzalez Herráez and L. Thévenaz, "Observation of pulse delaying and advancement in optical fibers using stimulated Brillouin scattering," Opt. Express 13, 82-88 (2005).

2 J. E. Sharping, Y. Okawachi, A. L. Gaeta, "Wide bandwidth slow light using a Raman fiber amplifier", Opt. Express 13, $6092-6098$ (2005).

3 D. Dahan and G. Eisenstein, "Tunable all optical delay via slow and fast light propagation in a Raman assisted fiber optical parametric amplifier: a route to all optical buffering," Opt. Express 13, 6234-6249 (2005).

4 K. Y. Song, M. Gonzalez Herraez, L. Thévenaz, "Gain-assisted pulse advancement using single and double Brillouin gain peaks in optical fibers," Opt. Express. 13, 9758-9765 (2005)

5 M. Gonzalez Herraez, K. Y. Song, L. Thévenaz, “Arbitrary-bandwidth Brillouin slow light in optical fibers,” Opt. Express 14, 1395-1400 (2006)

6 Z. Zhu, A.M.C. Dawes, D.J. Gauthier, L. Zhang, and A.E. Willner, '12-GHz-Bandwidth SBS Slow Light in Optical Fibers,' postdeadline paper, in OFC/NFOEC 2006 Proceedings Series (Optical Society of America, Washington, D.C., 2006), Anaheim, CA, Mar. 5-10, 2006. 